\title{
Quantum Gravity and Particle Physics
}

\section{Michael B. Green, London}

(Queen Mary College, University of London)

Until relatively recently it was commonly assumed by particle physicists that the force of gravity could be ignored in considering the structure of the elementary particles and the nature of the forces between them. However, the natural energy scale in any theory that tries to unify the electroweak and strong forces (the so-called "Grand Unified Theories" (GUTs) is close to the Planck energy $\left(10^{19} \mathrm{GeV}\right)$. This is the scale at which the quantum mechanical effects of the gravitational force are uncontrollably strong. It is just at the Planck scale that the Heisenberg uncertainty principle combined with general relativity implies that the quantum fluctuations are big enough to create microscopic black holes. The structure of all field theories must therefore be reconsidered on these scales and the quantum mechanical effects of the gravitational force should be important.

The present flurry of excitement over superstring theories arises from the fact that they are particle theories that embody general relativity in a manner which is consistent with quantum mechanics. The structure of these theories is quite novel, representing a radical departure from conventional quantum field theory which is the framework of most earlier ideas in particle physics. Whereas in all conventional quantum field theories the constituent particles (the electron, photon, quarks, ...) are pointlike, the fundamental constituents of superstring theories are extended strings which can vibrate as they move in space-time. The constraints on constructing consistent string theories are very profound and lead to an almost unique prediction of how the non-gravitational forces are embedded in the theory. In other words, requiring the quantum consistency of the theory leads to an almost uniquely prescribed prediction of a unification of the forces with few arbitrary parameters.

A single string can vibrate in any combination of an infinite number of normal modes of unlimited frequency. In quantum mechanics these modes corres- pond to states of definite energy and hence mass. The spectrum of the theory is therefore very rich, consisting of an infinite number of states with unlimited masses. The ground states in a superstring theory are massless - the word 'super' referring to the fact that these theories contain supersymmetry, a symmetry relating fermions to bosons.

The spacing of the excited, massive states is determined by the string 'tension', $T$, which has dimensions of [mass $]^{2}$ in natural units. This sets the scale for dimensions in the theory and should, therefore, be somewhere around the Planck value of $\left(10^{19} \mathrm{GeV}\right)^{2}$. The massive particles are therefore extremely massive and could never be directly produced in accelerators. At energies much smaller than $10^{19} \mathrm{GeV}$ only the massless modes are important for many considerations.

Among the massless states of the string are some spin 1 particles which are just the gauge particles of Yang-Mills theories and a massless spin 2 particle which is the graviton of general relativity. In addition, the supersymmetry of superstring theory is correlated with the existence of the spin $3 / 2$ 'gravitino' in the spectrum. The existence of these familiar massless particles is related to the fact that at low energy, when the massive states are irrelevant, superstring theory looks like some conventional theory of gravity interacting with a gauge theory. All the observed elementary particles (quarks, leptons, gauge particles, ...) should emerge from among the massless modes, since even the heaviest of them is massless relative to the Planck scale. However, the existence of the excited string states plays a crucial rôle in the theory since they can be excited just at the scale where the problems with quantum gravity arise, around the Planck scale. At this scale superstring theory differs radically from Einstein's theory, or indeed, all conventional quantum field theories, which have pointlike constituents.

There are two stages in the study of the dynamics of superstrings. The first is to formulate the quantum mechanics of a single free string. In the second stage the interactions between strings must be taken into account.

\section{Single String Quantum Mechanics}

The classical behaviour of a free string moving in some arbitrary space-time background is determined by a minimum action principle, in which the action is taken to be the area of the sheet swept out as the string moves (the 'worldsheet'). The fact that the area is a geometrical quantity which is independent of any particular parametrization of the world-sheet, is crucial for the consistency of the theory. This principle generalizes the familiar one for a point particle, where the action is the length of the world-line swept out as the particle moves. The passage to a quantum mechanical theory only maintains this geometrical feature under very special conditions. For the simplest theory, the dimension of space-time must take a 'critical' value of 26 while for superstring theories it must be ten!

The idea that space-time might have extra dimensions is not a new one but the fact that the number of these extra dimensions is determined by the theory is quite novel. Clearly, in order to explain observed physics the extra six dimensions of superstring theories must be 'curled up' into an unobservably small size. In the end the nature of space-time must be determined by the theory itself since the theory contains general relativity - the equations of the theory should therefore determine that the extra dimensions do curl up.

\section{Interactions Between Strings}

In order to take interactions between strings into account properly one ought to formulate something like a quantum field theory of strings. In conventional field theories point particles arise as quantized disturbances in fields which are defined over all points in space-time. This suggests that there should be some kind of generalization to fields which are defined not on the space of spacetime points, but on the space of all possible string configurations. An individual string would then be a quantized 'wave' in this field. This involves a very deep generalization of the notion of space-time which is the subject of a lot of the current research. Once such a formulation of string theory is achieved the manystring effects associated with the creation and annihilation of strings will be automatically incorporated into the theory. So far this deep understanding of string theory is incomplete. At present the calculation of string interactions can 
only be carried out in the context of 'perturbation theory', using diagrams that are analogous to the Feynman diagrams that are used in conventional field theories based on point particles.

\section{Quantum-Mechanical Consistency}

One signal of the problem of wedding general relativity and quantum theory is the fact that such perturbation theory diagrams for Einstein's theory of gravity are plagued by infinite results. In superstring theory the answers are sensibly finite, but only for very special versions of the theory. In the most interesting kind of superstring theory, known as the heterotic theory, the requirement that the theory be finite restricts the possible symmetry groups that describe the nongravitational forces to just two possibilities $-\mathrm{SO}(32)$ and $\mathrm{E}_{8} \times \mathrm{E}_{8}$. At this point I am describing the results obtained before the curling-up of the extra six spatial dimensions is considered, so that all ten space-time dimensions are on an equal footing. Achieving a finite quantum theory containing gravity is an unprecedented success.

There is another aspect of this quantum-mechanical consistency which arises from the fact that the interesting superstring theories are those which are 'chiral', i.e., those which distinguish left and right handedness. The reason that these are the physically interesting theories obviously stems from the observed left-right asymmetry of the weak force. However, there is a grave danger that any chiral theory will develop inconsistencies, known as 'anomalies', due to quantum mechanical fluctuations. These anomalies represent a breakdown of important conservation laws which are built into the classical (i.e., nonquantum mechanical) formulation of the theory. These quantum fluctuations are described by the Feynman diagrams of perturbation theory beyond the most trivial order. Requiring the absence of anomalies imposes severe conditions on superstring theories which again restricts the symmetry groups that unify the forces to those mentioned above.

These unifying symmetry groups represent far larger symmetries than those that are usually suggested in GUTs, which are attempts to unify the electroweak and the strong forces by simply postulating some symmetry. However, the striking fact is that all the plausible GUTs are based on symmetry groups which are subgroups of $E_{8}$ (which has very special properties and has long been suggested as an attractive group

\section{Huge Success for a Non-Standard Model}

The European Physical Society is pleased to announce the outstanding success of the first year's operation of

\section{Europhysics Letters}

now appearing with a new front cover.

So great has been the response by authors to the appearance of Europhysics Letters, the number of pages has had to be increased considerably. Number 1 of Volume 3 has been published with 128 pages and it will be followed by a series of bumper issues to keep pace with the inflow.

The Co-editors and the Management Board are determined to limit as far as possible the time between acceptance of contributions and publication and are not allowing cost considerations to limit output. Nor will there be a change in subscription price in 1987 from that announced, and all subscribers will benefit from this policy.

Libraries not yet receiving Europhysics Letters should take advantage of the exceptional value of the present offer.

Subscription for a full year - 24 issues in two volumes for only Sw.Frs. 590.-.

Send your order to: Les Editions de Physique

Av. du Hoggar - Z.I. de Courtaboeuf

B.P. 112 F-91944 Les Ulis Cedex

Europhysics Letters is published under the scientific responsibility of the European Physical Society.

\section{A RECENT CO-PUBLICATION}

\author{
of
}

Monotypia Franchi

06012 Città di Castello (PG), Italy and

Office for Official Publications

of the European Communities

Luxembourg

PROCEEDINGS OF THE COURSE AND WORKSHOP ON

\section{Basic Physical Processes of}

Toroidal Fusion Plasmas

held at the

INTERNATIONAL SCHOOL OF

PLASMA PHYSICS

«PIERO CALDIROLA »

Varenna, Italy

August 26 - September 3, 1985

\section{EDITORS}

\section{G.P. LAMPIS and M. LONTANO Istituto di Fisica del Plasma del CNR Euratom - CNR Association MIL.AN, Italy}

G.G. LEOTTA and A. MALEIN Commission of the European Communitice Directorate-General XII - Fusion Programme BRUSSELS. Belgium

E. SINDONI

Department of Physics. University of Milan MIL.AN. Italy

The Status of Toroidal Fusion Plasma Experiments and Theory. The subject matter of the Course and Workshop includes Tokamak experiments, Transport and Stability. Theory of Equilibrium, Stability and Transport in Stellarators, Nonaxisymmetric Toroidal Confinement experiments, MHD Stability of General Toroidal Confinement Systems, Resistive Processes and Boundary Phenomena in Toroidal Confinement, Beta-limits, Fusion Processes in Toroidal Confinement Systems (e.g.. Alpha-Particle Effects, Ignition Criteria in Toroidal Confinement), Perspective for an Ignition experiment. Topics in Confinement Analysis of Tokamaks with Auxiliary Heating. Plasma Heating in Toroidal Systems . Microinstabilities and Anomalous Transport in Toroidal Confinement Systems.

Emphasis is laid on : The latest results from Garching on $\mathrm{H}$-modes in ASDEX and Stellarator experiments; Confinement in the Bumpy Torus; Results from TFTR, JET and JT-60: Bean-Shaped Configurations in PBX: Sawtooth behaviour in Tokamaks, in particular in FT and TFTR; Charge-Exchange measurements on Doublet III ; Impurity measurements in TEXTOR; Pellet fuelling in the Alcator C Plasmas; Computer predictions of ATF physical results; State of the art of Ignitor machine.

Two volumes $\simeq 750 \mathrm{pp}$ September 1986

Price: ECU 83 ; BFR 3600, LIT 115.800 .

UKL 57.60. USD 81

Please order from MONOTYPIA FRANCHI

Bank Account No. 20370-83 at the Umbertide Agency of the Cassa di Risparmio di Perugia. Italy (SWIFT CR PG IT 3P).

Only the participants at the 1985 Varenna School will be entitled to a complimentary set of the Procecdings 
for particle physics). For this reason the superstring theory with $E_{8} \times E_{8}$ is a particularly interesting candidate for providing a realistic theory. An immense amount of research has been aimed at deriving the physical predictions from this theory. The most essential point is that when the curling-up of the six extra space dimensions is considered, the huge symmetry, $E_{8} \times E_{8}$ breaks down to a subsymmetry. The result is a theory in effectively four space-time dimensions in which there is a kind of GUT symmetry unifying the non-gravitational forces. Since the starting point is a superstring theory with no freely adjustable parameters there are few parameters in this unifying theory (whereas a typical GUT has around 25 parameters).

\section{Curled-up Dimensions}

In principle the equations of the theory determine the dynamics of the theory with curled-up dimensions. As I have indicated above, string theory is not yet formulated in a satisfactory manner and the most profound problems cannot yet be faced. However, there has been a lot of progress in understanding the restrictions that the theory places on the nature of the curled-up space. In contrast to the theories of quantum gravity based on pointlike particles, in string theory there are very few possible ways in which the extra six dimensions can be curled up.

Although there are strong theoretical restrictions on the extra six-dimensional space there is not, at the present level of understanding, any theoretical reason for selecting one rather than another. Any of these seems to result in an equally good effectively four-dimensional (three space and one time) theory so that at this stage there is some freedom to appeal to experiment to constrain the possibilities. It is a matter of some debate whether the theory should predict one particular version of the curled-up space or whether there is some statistical uncertainty about which of the many possible theories with six curled-up (and four effectively flat) dimensions Nature has chosen. For most of the possibilities so far discovered the consistency of the theory determines that the original $E_{8} \times$ $\mathrm{E}_{8}$ symmetry of the ten-dimensional theory is broken down to $E_{6} \times E_{8}$, although there is also a possibility that it may be broken to the group $S O(10) \times E_{8}$ or $S U(5) \times E_{8}$. These are all very interesting symmetries for the theory at very high energies since the groups $S U(5), S O(10)$ and $E_{6}$ have all been suggested earlier as plausible GUT symmetries. The extra factor of $E_{8}$ sym- metry is an added bonus. The matter carrying the $\mathrm{E}_{8}$ charges is neutral with respect to the matter we observe, and would only be detectable via its gravitational force. It has been called 'shadow matter'. Detailed properties of this type of matter are not understood - it is not even known, for example, whether this extra $E_{8}$ symmetry remains intact or is broken. Whether any shadow matter survives in the Universe depends on illunderstood details of the early evolution of the Universe.

Many of the properties of the effective theory in four dimensions are determined entirely by topological features of the six curled-up dimensions. This is a very striking feature of superstring theories which is not shared by earlier theories that invoked extra dimensions. For example, the number of 'generations' of quarks and leptons (i.e., the number of different species) is determined by one of the most fundamental topological properties of the space, namely, its Euler characteristic. There are reasons for believing that in a theory of this type there can be at most four generations. This provides a severe restriction on the possible curled-up spaces.

\section{Limitations}

Although the $E_{8} \times E_{8}$ superstring theory has an excellent chance of leading to a unified qualitative explanation of accelerator experiments it is still a very long way from providing a detailed description of experimental features. The only calculations that have been performed are in the context of perturbation theory and going beyond this will require the understanding of several formidable theoretical problems. There are, however, strong arguments that suggest that such non-perturbative features must be essential. For example, in most versions of the theory, supersymmetry is unbroken to all orders in perturbation theory. Since supersymmetry is very obviously broken in the real world it is important to understand the non-perturbative mechanisms that break it. Whereas unbroken supersymmetry ensures that all the observed particles have zero mass relative to the Planck scale, the breaking of supersymmetry will lead to the actual non-zero masses for the observed particles and for newly predicted particles (such as the supersymmetric partners of the known particles). Since these kinds of detailed predictions are difficult to obtain convincingly from the theory it is important to emphasize the qualitative features that distinguish superstring theory from more ad hoc GUTs. Among these kinds of features are the existence of shadow matter and of low energy symmetries among particles beyond those of the standard model as I have already mentioned. Another intriguing possibility is the existence of very heavy particles with peculiar fractional electric charges. These are associated with states in which a string winds through the holes in the curled-up six-dimensional space. If these states exist then there should also be magnetic monopoles with magnetic charges which are multiples of the basic unit of magnetic charge. Unfortunately, none of these unusual phenomena is likely to be measureable for a long time.

There is one striking experimental fact that must be explained if superstring theory is to make physical sense. This is the fact that the 'cosmological constant' - a measure of the part of the curvature of the universe that is not due to the presence of matter - is measured to be almost exactly zero whereas a typical point-particle theory gives a value close to unity! Since the typical point-particle theory does not contain quantum gravity in a consistent manner this problem has often been swept under the same rug as other inconsistencies. However, since superstring theory contains quantum gravity in a consistent manner, the explanation of the vanishing (or near vanishing) of the cosmological constant might prove to be a key test for the theory.

It is could well be the case that a deeper understanding of the predictions of the theory will have to await a more fundamental formulation that I alluded to earlier. Instead of general relativity emerging from the theory in an apparently accidental manner there is presumably a deep principle in string theory that extends the usual geometric principles at the heart of general relativity and Yang-Mills gauge theories. This principle is expected to involve geometrical notions in the space defined by all the possible configurations of a string - a vastly larger space than the conventional view of space-time leven in ten dimensions!). A lot of the current research is aimed at discovering this generalization of general relativity. Such a reformulation of the theory ought to clarify its predictions and point to the useful kinds of experiments that could test the theory.

\section{FURTHER READING}

1. Green M.B., Nature 314 (1985) 409-414.

2. Ellis J., Nature 323 (1986) 595-598.

3. Green M.B., Schwarz J.H. and Witten E., Superstring Theory (in two volumes) (Cambridge University Press) 1986. 